\title{
IMPACT OF USERS' MOTIVATION ON GAMIFIED CROWDSOURCING SYSTEMS: A CASE OF STACKOVERFLOW
}

\author{
Sarah Penoyer, Georgia College, sarah.penoyer@bobcats.gcsu.edu \\ Breana Reynolds, Georgia College, breana.reynolds@bobcats.gcsu.edu \\ Bryan Marshall, Georgia College, bryan.marshall@gcsu.edu \\ PeterW. Cardon, University of Southern California, petercardon@marshall.usc.edu
}

\begin{abstract}
Companies like StackOverflow use crowdsourcing and gamification to entice users to participate in the answering of questions in the online forums. StackOverflow has overwhelmingly become the go to place for users to seek help with programming related questions. This research aims to investigate what factors influence the users on StackOverflow to participate. We survey 200 of the highest ranked users on StackOverflow. We asked them questions related to both extrinsic and intrinsic motivations. Surprisingly, we found that for the highest ranked users on StackOverflow intrinsic motivations like helping others, reciprocity, and making an impact are more important than financial gains and organizational pressures.
\end{abstract}

Keywords: crowdsourcing, gamification, software development, forums, help desk

\section{INTRODUCTION}

Gamification, a term coined in 2010, is making great headway in the professional world. The most frequently cited definition of gamification is "the use of game design elements in non-game contexts" (Deterding, Dixon, Khaled, \& Nacke, 2011). Thus, it applies gaming mechanics to create both intrinsic and extrinsic motivations to support target behaviors by users (DuVernet and Popp, 2014). It is used "to measure, influence and reward target user behaviors" (Maan, 2013). Gamification is being used in companies for many reasons, including for training programs, hiring assessments, sales incentives programs, and customer service initiatives (DuVernet and Popp, 2014).

The purpose of this paper is to identify the motivations of top users on StackOverflow, a gamified system of crowdsourcing. StackOverflow utilizes practices of gamification and crowdsourcing on its platform. Users with specific interests and questions share their experiences on the platform. Users are assigned a reputation ranking that signifies their expertise in the information technology field, prestige, and overall competence. As a public and open forum, StackOverflow appeals to users for its wealth of knowledge and the opportunity for any user to share and receive knowledge, thus providing a valuable, open resource to everyone.

While a lot of research has addressed gamification, it has focused primarily focused motivations to drive participation in early stages of adoption or early stages of initiatives. As a result, little is known about the motivations of long-term users. Our study addresses the degree to which extrinsic and intrinsic motivational factors influence long-time users of gamified crowdsourcing platforms. This is particularly important since it focuses on the long-run success of gamified systems.

\section{LITERATURE REVIEW}

Literature on how gamification and crowdsourcing influence behavior is abundant; however, the research on perceptions and actual motivations of users on gamified software crowdsourcing platforms is limited. Rather, most research focuses on consumer-based online forums. For example, according to a study, more than $40 \%$ of consumers turn to online consumer communities for help with products, resulting in more than a $15 \%$ reduction in service cases and support costs for firms (Thompson et al, 2016). 
Studies of gamification have shown users are more engaged, more interested in learning, and appear to participate heavily when there is public visibility of contributions. For example, a recent study showed that gamification provided richer data and increased participant engagement (Bailey \& Pritchard, 2015). In addition to increased engagement, studies have shown users are driven to learn more (Yang et al, 2017). The public visibility of these rewards appears to influence user behavior. A recent study showed then when contributions and rewards could be seen among everyone, there was higher user engagement (Garcia et al, 2017). This would lead us to believe that participants on the StackOverflow site may be sensitive to their answers and lead to greater engagement with the people asking the questions.

Software discussion forums, such as StackOverflow, create the ideal landscape for crowdsourcing. It has galvanized a community of collaborators and knowledge seekers, which according to their website, "is the largest most trusted online community for developers to learn, share their knowledge, and build careers. More than 50 million professional and aspiring programmers visit Stack Overflow each month to help solve coding problems, develop new skills, and find job opportunities" (StackOverflow, 2018).

Gamification has been widely used on forum sites such as StackOverflow with rewards such as badges, stars, awards, and rankings for active participation of sharing information publicly. In many cases these forum sites rely on crowdsourcing, which is the ability to leverage internal resources by gathering consumer information to expand into external networks by empowering members to create value, rather than simply being consumers (Kohler, 2018). Overall, the advantage to crowdsourcing communities is the ability to collectively generate digital information products, services, or knowledge (Khansa et al, 2015).

In recent years, gamified applications have been carried over into companies in nearly all industries. Within the workplace, a common form of gamification is badges. Kidwell (2016) states, "Badges are an easy means of signaling and incentivizing desirable behaviors," and leaders of businesses are recognizing how to further motivate their employees to not only learn new tasks but maintain their knowledge on what they know already to keep them engaged in seeking ways for promotions and overall career success. One of the primary elements of gamification is the badges and leaderboards that are embedded within the software discussion application. These badges, rewards and public recognition stand as motivation for sharing of knowledge publicly on forums. However, "[g]amification is a recent trend that offers to increase engagement through the inclusion of game-like features like points and badges in nongame contexts," and these practices are now being carried over to corporations to further improve motivation among employees within the workplace with concepts of a game with a winning factor (Looyestyn, 2017). The school of Health Sciences in the University of Australia conducted 15 surveys and 12 out of the 15 surveys provided evidence of positive significant effects in favor of gamification (Looyestyn, 2017).

Collaboration among users on crowdsourced platforms is driven by many motivations, including intrinsic and extrinsic, to further one's own knowledge, to change behavior in a workplace, or to better a business process or output (Khansa et al, 2015). Scholars have debated whether gamification, which awards badges and ranking titles, are primarily driven by intrinsic or extrinsic motivations. Regarding intrinsic motivations, "[t]he goal of meaningful gamification ... is to help users find meaningful connections with the underlying non-game activities, and using rewards only when truly necessary," so the use of badges and rankings does help, but taking these rewards away shouldn't take away motivation as a whole if there are meaningful connections made among the users (Nicholson, 2013). Extrinsic motivation cannot be seen as controlling behavior because "people integrate external motivation in their sense of self," and the practice of gamification aids in hiding that aspect of control with the use of gaming concepts, such as rewards and high titles (Nicholson, 2013). This school of thought suggests extrinsic motivation may be useful in the beginning to increase participation; however, intrinsic motivation becomes the driving force as their sense of self over time focuses on user satisfaction. Yet, everyone perceives success differently; therefore, the practice of gamification may be driven by diverse extrinsic and intrinsic motivations by various users. What's clear based on market research studies is that "gamification can provide richer data and increase participant engagement." (Bailey \& Pritchard, 2015).

One of the primary unaddressed issues of past research on gamified crowdsourced platforms is the long-term motivations of users. The research has neglected the importance of habit-forming behaviors that are a result of gamification being implemented within software discussion forums that have active professional users outside of the digital community. This lack of research is because the short-term effect of badges and rewards is observed in the 
initial rewards and better-quality output, but long-term motivation is less obvious and observable as it becomes a pattern-formed habit. Thus, the excitement of badges and potential rewards may become less important over time. This research is to examine long-term user behavior in gamified environments. We hypothesize that long-term users will be primarily motivated by intrinsic motivations.

\section{RESEARCH QUESTIONS}

The purpose of this research is to identify the motivations behind long-term users of the gamified crowdsourcing platform StackOverflow. Specifically, we addressed the following questions:

1. To what extent do organizations influence top users on StackOverflow to seek high reputation scores?

2. To what extent do financial rewards influence top users on StackOverflow to seek high reputation scores?

3. To what extent does an increase in potential professional opportunities influence top users on StackOverflow to seek high reputation scores?

4. To what extent does helping others or altruism influence the behavior of top users on StackOverflow to seek high reputation scores?

5. To what extent does the feeling of making an "impact" influence the behavior of top users on StackOverflow to seek high reputation scores?

6. To what extent does the feeling of "reciprocation" or returning the favor influence the behavior of top users on StackOverflow to seek high reputation scores?

\section{METHODOLOGY}

To understand the long-term motivations of users on gamified crowdsourcing platforms, we specifically focused on top users on StackOverflow who have used it for an extensive period of time. We identified the power users on StackOverflow by reputation. These reputation scores are publicly available and can be sorted based on all-time reputation.

StackOverflow allows for an abundance of information on their users, especially if they have taken the time to set up their profile. For example, the number one user on StackOverflow is Jon Skeet (StackOverflow, 2018). Jon's profile says he works at Google as an engineer and has reached almost 235 million people with his answers. He has asked 50 questions and has answered 34,139 times. He has been a member for 9 years 7 months (or 115 months). This equates to about almost 300 answers a month or about 10 a day. He also lists his GitHub account, which then displays his email address.

We sent top StackOverflow users a custom email (Appendix A) asking them to fill out a simple survey (Appendix B) The survey instrument consisted of 13 questions. The first 5 questions pertain to their professional background. Question 6 was about their about their profile on StackOverflow. The next question was about motivations, both intrinsic and extrinsic. Question 8 and 9 surveyed them on their business communication skills and where they displayed their reputation on the web. The last three questions were about demographics.

To find top users, we scoured the first 35 pages of users and found 191 email addresses. We sent out 191 emails and had a $23 \%$ response rate. Of the 45 respondents, all of them were male. Over $50 \%$ of the respondents reported they have a graduate degree, while $27 \%$ reported they earned an undergraduate university degree, and $20 \%$ only have a high school diploma. All but one reported full-time employment, with $20 \%$ of them as independent contractors, freelancers, or self-employed. Respondents were from the following countries: USA (17), UK (6), Germany (3), Canada (3), France (2), India (2), Austria (1), Bangladesh (1), Denmark (1), Israel (1), Italy (1), Nepal (1), Netherlands (1), Russia (1), Sweden (1), and Switzerland (1).

As far as how long they have been working with StackOverflow, 42 users reported they have been using it for an average of 8 years 1 month. Most these top users joined StackOverflow at its launch, when StackOverflow was still in "preview" mode. The average respondent had a reputation of 181,395, with the highest reputation of 1,022,218. 
In StackOverflow, users are given "badges" when they accomplish certain tasks. There are different levels of badges, some easier to earn then others. An example of a "gold" badge is the Socratic Badge. This badge is earned if a user has a well-received question on 100 separate days and maintains a positive question record. The overall total badges earned by the surveyed users amounted to 45,556 or 1,111 each. Of the 44 respondents, they had earned a total of 2,288 gold medals, with an average of 54 each.

One of the demographics reported was reach, which is the number of views each of the answered questions received. With the 40 people that answered this question on the survey, they had a combined reach of 956,000,000 or 25,000,000 on average per user. This is a pretty powerful metric. Reach speaks directly to the relevance of questions and answers and how impactful they are to the community.

Roughly 93 percent of the respondents reported they work as professional developers. The average length of work experience was 15 years. The 41 respondents reported a total of 616 years of professional developer experience. $85 \%$ reported they code as a hobby outside of work. Presumably the best programmers in the world code as a hobby outside of work.

\section{RESULTS}

We grouped our findings into three areas: extrinsic motivation, intrinsic motivation, and public display of reputation rankings.

\section{Extrinsic Motivation}

We grouped extrinsic motivation into two categories: (a) organization and (b) individual. As shown in Table 1, respondents perceive little interest in extrinsic motivation from their organizations. Just 7 percent of users felt rewards from their organizations were important, and just over 2 percent thought it was important to raise the reputation of their organizations. We felt this would have been higher, especially since $20 \%$ of the users reported either working independently or owning the company.

Further, we found that users report little extrinsic motivation for individual rewards. No respondents $(0 \%)$ thought being financially compensated was important. Just 14 percent of users perceived additional opportunities was important. About a quarter (26\%) of users valued receiving a higher professional reputation.

Table 1. Extrinsic Motivation Levels of Top StackOverflow Users

\begin{tabular}{|c|c|c|c|}
\hline Survey Item & $M$ & $S D$ & $\begin{array}{l}\text { \% Consider } \\
\text { Important }\end{array}$ \\
\hline \multicolumn{4}{|l|}{ Extrinsic Motivation -- Organization } \\
\hline My organization rewards me for having a high reputation score. & 2.18 & 1.74 & 6.82 \\
\hline It raises the reputation of your organization. & 1.48 & 1.27 & 2.27 \\
\hline \multicolumn{4}{|l|}{ Extrinsic Motivation - Individual } \\
\hline I am financially compensated for having a high reputation score. & 1.09 & 0.47 & 0.00 \\
\hline I gain additional professional opportunities due to a high reputation score. & 3.68 & 1.75 & 13.64 \\
\hline It raises the value of my professional reputation. & 4.67 & 1.49 & 25.58 \\
\hline
\end{tabular}

Note. Each of the survey items was based on a Likert scale from $1=$ not important to $7=$ extremely important. The percentage of respondents who considered an item important (final column in this table) is based on the number of respondents who selected 6 (important) or 7 (extremely important) on the Likert scale for that item. 


\section{Intrinsic Motivation}

We found that respondents are much more likely to report intrinsically motivating factors as important (see Table 2). In particular, 84 percent of respondents say they love helping others. Just over 61 percent report that making an impact is important to them. Finally, about 36 percent report that helping others is important because other people have helped them. With so much experience and expertise, these high-end users' question-to-answer rate is very low. This likely influences the belief that much of their contributions are not reciprocal in nature, since they help others much more than they get help.

Table 2. Intrinsic Motivation Levels of Top StackOverflow Users

\begin{tabular}{|l|c|c|c|}
\hline Survey Item & $\boldsymbol{M}$ & $\boldsymbol{S D}$ & \% Consider Important \\
\hline I love helping others. & 6.39 & 0.81 & 84.09 \\
\hline I feel I can make an impact. & 5.86 & 1.07 & 61.36 \\
\hline I help others because people help me. & 4.59 & 1.74 & 36.36 \\
\hline
\end{tabular}

Note. Each of the survey items was based on a Likert scale from $1=$ not important to $7=$ extremely important. The percentage of respondents who considered an item important (final column in this table) is based on the number of respondents who selected 6 (important) or 7 (extremely important) on the Likert scale for that item.

\section{Display of Reputation Scores}

While top users report that extrinsic motivation is generally not important to them, they do display their reputation scores outside of StackOverflow in a variety of places (see Table 3). The average users in our survey displayed this badge in 1.13 places. The most common place they display their rankings are in their resumes, LinkedIn pages, and personal webpages. Interestingly, very few of these top users post their rankings on social networking sites such as Facebook or Twitter.

Table 3. Public Displays of Reputation Scores by Top StackOverflow Users

\begin{tabular}{|l|c|c|}
\hline Place top users post StackOverflow reputation scores & $\#$ & \% \\
\hline Resume or curriculum vitae & 18 & 40.0 \\
\hline LinkedIn or other professional sites & 12 & 26.7 \\
\hline Personal web page or personal blog & 11 & 24.4 \\
\hline GitHub repository & 5 & 11.1 \\
\hline In presentations & 4 & 8.9 \\
\hline Email signature line & 2 & 4.4 \\
\hline Social networking sites (Facebook, Twitter, etc.) & 1 & 2.2 \\
\hline
\end{tabular}

Note. Based on 45 respondents.

\section{CONCLUSIONS}

The biggest take away from this research paper has to be that motivation is important and that gamification appears to have some influence on that motivation (Nicholson, 2013). Previous research reported that although the initial motivation may be extrinsic, that if the user finds an intrinsic motivation they typically stick with the process longer. This research has confirmed this emphatically. Long term users on StackOverflow have found the intrinsic motivations to keep them going and helping others. Future research is needed to further expand on this phenomenon.

\section{REFERENCES}

Bailey, P. \& Pritchard, G. (2015). Gamification in Market Research. The Market Research Society, 57(1). 17-27. 
Deterding, S., Dixon, D., Khaled, R., \& Nacke, L. (2011). From game design elements to gamefulness: Defining "gamification." In Proceedings of the 15th International Academic MindTrek Conference: Envisioning Future Media Environments (pp. 9-15). New York, NY: ACM. https://doi.org/10.1145/2181037.2181040

StackOverflow. (2018) Insights Survey. https://insights.stackoverflow.com/survey/2018/

Kidwell, M. C., Lazarević, L. B., Baranski, E., Hardwicke, T. E., et al (May 12, 2016). Badges to Acknowledge Open Practices: A Simple, Low-Cost, Effective Method for Increasing Transparency. Journal of PLOS Biology, 14(5), 1-16.

Khansa, L., Ma, X., Liginlal, D., \& Kim, S. (2015). Understanding Members’ Active Participation in Online Question-and Answer Communities: A Theory and Empirical Analysis. Journal of Management Information Systems, 32(2), 162-203.

Kohler, T. (2018). How to Scale Crowdsourcing Platforms. California Management Review, 60(2), 98-121.

Maan, J. (August 2013). Social Business Transformation through Gamification. International Journal of Managing Information Technology, 5(3), 9-16

Looyestyn, J., Kernot, J., Boshoff, K., Ryan, J., Edney, S., \& Maher, C. (March 31, 2017). Does gamification increase engagement with online programs? A systematic review. Looyestyn et al, 1-19.

Nicholson, S. (2013). Two Paths to Motivation through Game Design Elements: Reward-Based Gamification and Meaningful Gamification. iConference 2013 Proceedings. (pp 671-672).

DuVernet, A.M. and Popp, E. (2014) Gamification of Workplace Practices. The Industrial Organizational Psychologist, (52), 39-44.

García, O., Navarro, H., Rodríguez, eFerdández, D., \& Freixes, D. (2017). Dealing With Transformation in a VUCA World: A Competence-Based Development Project Applying Transmedia Storytelling and Gamification for an International Corporation, Cookie Box, S.L., Spain. 919-926.

Thompson, S., Kim, M., \& Smith, K. (April 2016). Community Participation and Consumer-to-Consumer Helping: Does Participation in Third Party-Hosted Communities Reduce One's Likelihood of Helping? Journal of Marketing Research, 53, 280-295.

Yang, Y., Asaad, Y. and Dwivedi, Y. (2017). Examining the impact of gamification on intention of engagement and brand attitude in the marketing context. Computers in Human Behavior, (73), 459-469. 


\title{
APPENDICES
}

Appendix A. Outbound Email Request

\begin{abstract}
My name is Sarah Penoyer, and I am a MIS student at Georgia College. Currently, I am conducting research on gamification and crowdsourcing. We recently saw that you are extremely highly ranked on StackOverflow as a top reputation user. With your combined knowledge of StackOverflow and technology, our hope was to collect data on your experience with the software forum.
\end{abstract}

Would you be interested in answering a few questions in relation to crowdsourcing and gamification on

StackOverflow? I've attached the survey below, and would appreciate any available answers.

https:/www. surveymonkey.com/r/stackoverflow-2018

Thank you in advance, and I hope you're having a great week!

Sarah

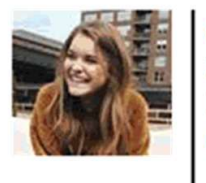

Sarah Penoyer

Management Information Student

E: sarah.penoyer@bobcats.gcsu.edu

Georgia College

Appendix B. Survey Questions

\section{Employment status:}

- Employed full-time

- Independent contractor, freelancer, or self-employed

- Not employed, but looking for work

- Employed part-time

- Not employed, and not looking for work

- Retired

2. Do you work as a professional developer?

- Yes

- No

3. If you responded "Yes" to the first question, how many years have you worked as a professional developer?

4. What developer roles do you hold? Check ALL that apply.

- Back-end developer

- Full-stack developer

- Front-end developer

- Mobile developer

- Desktop or enterprise applications developer

- Database administrator

- Designer

- System administrator

- DevOps specialist

- Other (please specify)

5. Do you code as a hobby outside of work? 
- Yes

- No

6. The following questions can be answered directly from your StackOverflow user profile page:

- How long have you been using StackOverflow?

- What is your current total reputation score?

- How many total badges do you have?

- How many "gold" badges do you have?

- What is your impact score?

7. How important are the following motivations for helping others on StackOverflow?

- It raises the reputation of your organization.

- My organization rewards me for having a high reputation score.

- I am financially compensated for having a high reputation score.

- I gain additional professional opportunities due to a high reputation score.

- It raises the value of my professional reputation.

- I love helping others.

- I feel I can make an impact.

- I help others because people help me.

8. How much do you agree with the following statements?

- I am very careful about the quality of my answers on StackOverflow because I worry about my rankings.

- I am very careful about the writing style (i.e., grammar, spelling) of my answers on StackOverflow because I worry about my rankings.

- I avoid being negative -- even when others ask bad questions -- because I worry about my rankings.

- I follow up with comments and questions because I want to be engaged in the conversation.

9. I display my StackOverflow reputation in the following places: (Check ALL that apply)

- LinkedIn or other professional sites

- Email signature line

- Social networking sites (Facebook, Twitter, etc.)

- Personal web page or personal blog

- Resume or curriculum vitae

- GitHub repository

- In presentations

- Other (please specify)

10. What country do you live in?

\section{Highest education level:}

- Graduate (Postgraduate) University Degree

- Undergraduate University Degree

- High School Diploma

12. Are you currently a student

- Yes

- No

13. Gender

- Woman

- Man

- Prefer not to say 